\title{
Fracturas del tercio medio facial. Experiencia en el Hospital Nacional Dos de Mayo, 1999 - 2009
}

\author{
Facial middle third fractures. Experience at Dos de Mayo National Hospital, 1999 - 2009
}

\author{
Francisco Avello Canisto ${ }^{1,2}$ \\ Servicio de Cirugia de Cabeza, Cuello y Máxilo-Facial del Hospital Nacional Dos de Mayo. \\ ${ }^{2}$ Departamento de Cirugia de la Universidad Nacional Mayor de San Marcos.
}

\begin{abstract}
Resumen
Introducción: Correspondiendo a una patología frecuente en la especialidad de cabeza, cuello y máxilo-facial, consecuencia de traumatismos severos, es importante determinar las caracteristicas de las fracturas del tercio medio facial, para su manejo. Objetivo: Determinar la presentación de las fracturas del tercio medio facial por edad, sexo, agente causal. Diseño: Estudio observacional descriptivo. Lugar: Servicio de Cirugia de Cabeza, Cuello y Máxilo-Facial del Hospital Nacional Dos de Mayo, Lima, Perú. Participantes: Pacientes con fractura del tercio medio facial. Intervenciones: Recolección de datos registrados en las historias clínicas, entre junio 1999 y mayo 2009. Principales medidas de resultados: Edad, sexo, agente causal y clasificación. Resultados: De 471 pacientes, $83 \%$ fue de sexo masculino, $72,5 \%$ tenía entre 21 y 40 años de edad, $48 \%$ fue por accidentes de tránsito y $25 \%$ por robos; $83 \%$ correspondió solamente a fracturas del tercio medio y $88 \%$ a trazo unilateral. Conclusiones: Las fracturas del tercio medio facial son las más frecuentes de la región facial; el diagnóstico clínico depende de la estructura afectada. Se debe actuar oportunamente para evitar secuelas y complicaciones.
\end{abstract}

Palabras clave: Tercio medio facial; complejo naso-maxilar; complejo máxilo-malar; complejo témporo-malar.

\section{Abstract}

Introduction: Facial middle third fractures resulting from severe trauma are frequent pathology attended by Surgery Head, Neck and Maxillofacial specialists; to determine its characteristics is important for treatment. Objectives: To determine the presentation of facial middle third fractures by age, sex, causal agent. Design: Observational descriptive study. Setting: Head, Neck and Maxillofacial Surgery Service, Dos de Mayo National Hospital, Lima, Peru. Participants: Patients with facial middle third fracture. Interventions: Collection of data from medical records June 1999 through May 2009. Main outcome measures: Age, sex, causing agent and classification. Results: From 471 patients, $83 \%$ were male, $72.5 \%$ between 21 and 40 years of age; $48 \%$ resulted from traffic accidents and $25 \%$ from robberies; $83 \%$ were middle third fractures only, and $88 \%$ unilateral stroke. Conclusions: Facial middle third fractures are the most common fractures of the facial region; clinical diagnosis depends on the affected structure. Prompt treatment is necessary to prevent sequelae and complications.

Key words: Facial middle third; naso-maxillary complex; maxillo-malar complex; temporo-malar complex.

An Fac med. 2013;74(2):123-8

\section{INTRODUCCIÓN}

Las fracturas del tercio medio facial corresponden a una patología frecuente dentro de la especialidad de cirugía cabeza, cuello y máxilo-facial, como consecuencia de traumatismos severos en la región, los mismos que pueden ser de diversa etiología. La determinación de este tipo de fractura, por edad, sexo y agente causal es importante para su manejo.

El tercio medio facial es una estructura ósea compleja; comprende principalmente a los maxilares superiores, huesos propios nasales, huesos malares y temporales. Se puede ver afectado por fracturas de trazo unilateral (más frecuentes) y de trazo bilateral (más complejas). El maxilar superior contribuye a la formación de estructuras, como las órbitas, fosas nasales, reborde alveolar y paladar.

Las fracturas de trazo unilateral las agrupamos en: a) complejo naso-maxilar, que comprende los huesos propios nasales y la apófisis ascendente del maxilar superior; b) complejo máxilomalar, que comprende al maxilar superior excepto su apófisis ascendente y la porción orbitaria del malar (reborde orbitario y apófisis marginal); y, c) complejo témporo-malar, que comprende a la apófisis cigomática del malar y del temporal. Teniendo en cuenta los tres complejos señalados, podemos clasificar las fracturas de trazo unilateral como sigue $^{(1)}$ (figura 1$)$ :

1. Fracturas del complejo naso-maxilar (pirámide nasal):

1.a. Huesos propios nasales.

1.b. Apófisis ascendente del maxilar superior.

2. Fracturas del complejo máxilo-malar: 2.a. Antral o pared anterior del maxilar.

2.b. Impactación malar. 


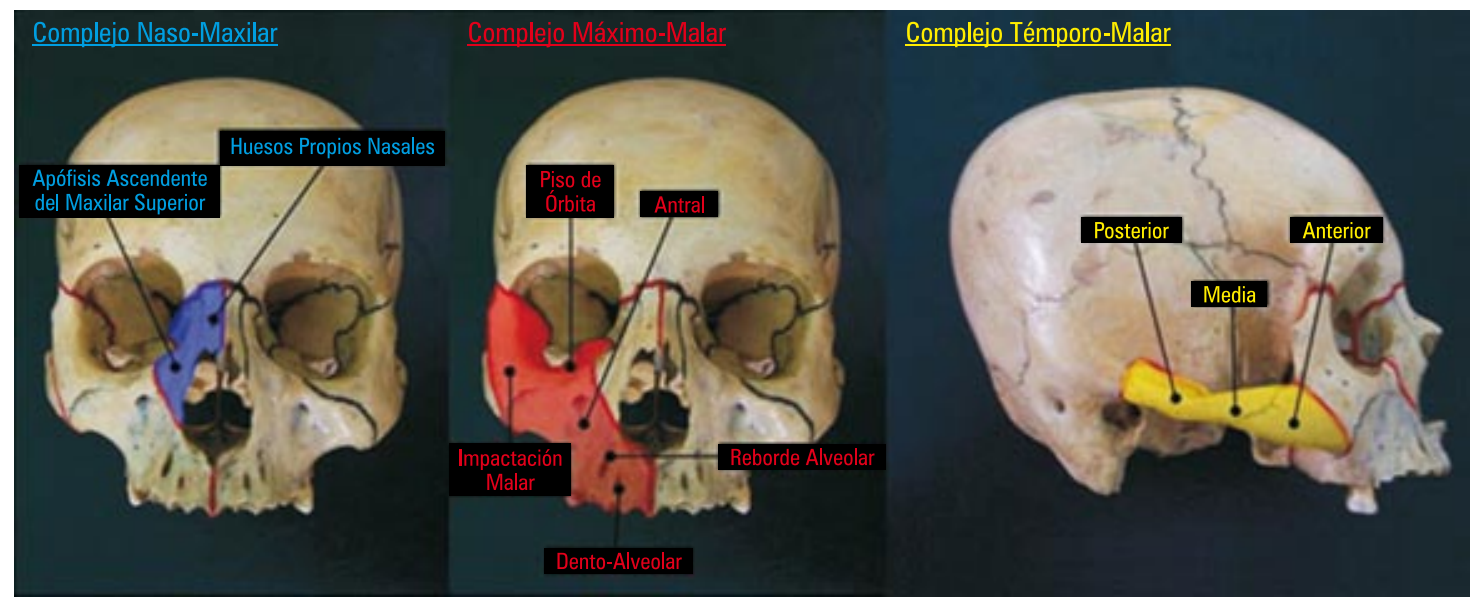

Figura 1. Se muestra los tres complejos del tercio medio facial que usamos para la clasificación de las fracturas de trazo unilateral, cada una con sus respectivos trazos de fractura. Complejo naso-maxilar de color azul, complejo máxilo-malar de color rojo y complejo témporo-malar de color amarillo.

\section{2.c. Piso de órbita (blow-out).}

2.d. Reborde alveolar (parcial o total).

\section{2.e. Dento-alveolar.}

3. Fracturas del complejo témporomalar (arco cigomático):

\section{3.a. Anterior o malar.}

\section{3.b. Posterior o temporal.}

\section{3.c. Media (en 'M').}

Estos tres complejos tienen relación directa con las zonas de trauma facial del tercio medio, delimitando de esta forma las estructuras óseas comprometidas y no cada hueso en forma aislada ${ }^{(1)}$.

El complejo máxilo-malar es el que se afecta con mayor frecuencia y dentro de este grupo tiene una incidencia mayor la impactación malar dentro del seno maxilar. La fractura antral o de pared anterior del maxilar no es frecuente verla aislada; por lo general, acompaña a la impactación malar y cuando se presenta en forma aislada puede no diagnosticarse y no recibir tratamiento, sin mayores problemas posteriores. La fractura del piso de órbita tampoco es frecuente verla aislada (blow-out); acompaña generalmente a la impactación malar. La fractura del reborde alveolar puede afectarlo total o parcialmente, desde incisivos hasta molares, movili- zando el segmento comprometido en bloque. La fractura dento-alveolar se produce generalmente por un traumatismo directo sobre una pieza dental, comprometiendo conjuntamente al hueso alveolar que la contiene, es decir, al movilizarse la pieza dental afectada se moviliza con el hueso alveolar que rodea su raíz (tabla interna - tabla externa) ${ }^{(1)}$.

En la producción de este tipo de fracturas se requiere la acción de un traumatismo de regular o mayor intensidad, generalmente producida por objetos contusos, durante asaltos o agresiones; pero, en la mayoría de los casos, las causas de estas fracturas son los accidentes de tránsito ${ }^{(2)}$. Las caídas en forma casual o por estado de ebriedad son causa importante, pero menos frecuentes. Se debe tener en cuenta las heridas por proyectil de arma de fuego y explosivos, como indicador del aumento de la violencia en nuestro país ${ }^{(2,3)}$.

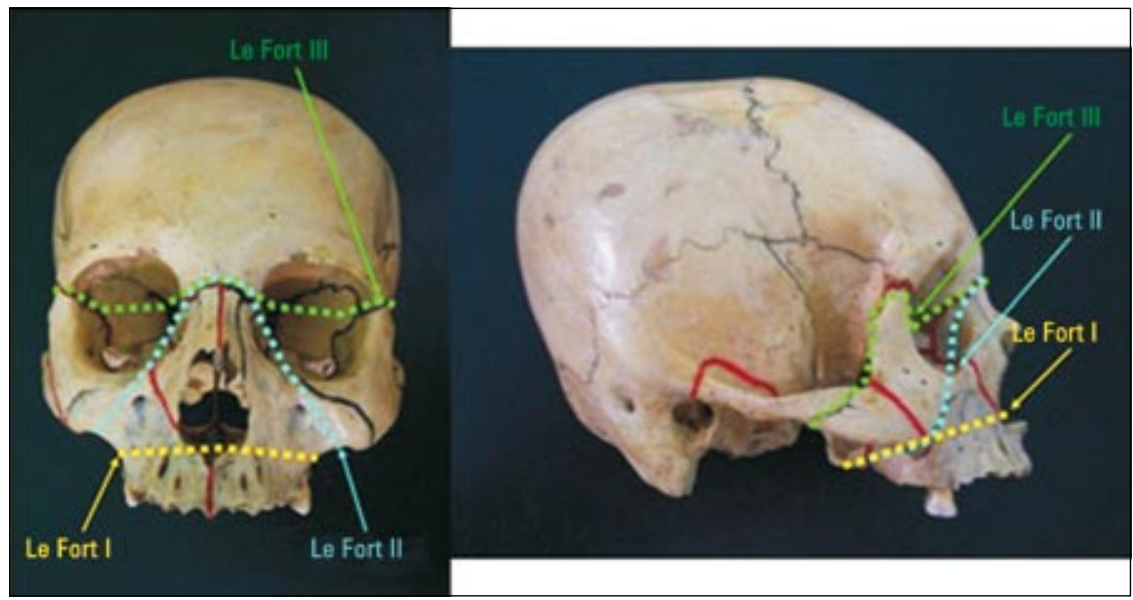

Figura 2. Se muestra los tres tipos de fracturas de Le Fort o fracturas de trazo bilateral del tercio medio facial; vista frontal y lateral. En la vista frontal y en segundo plano podemos ver al lado derecho divisiones en color rojo, que nos indican los límites entre los tres complejos óseos del tercio medio facial ya mencionados, mientras que al lado izquierdo vemos divisiones en color negro que nos indican los límites entre los huesos que lo conforman. 
Las fracturas de trazo bilateral del tercio medio facial son más complejas que las de trazo unilateral, pero menos frecuentes, aproximadamente $15 \%$ de todas ellas. Se las clasifica como sigue, y a su vez estas fracturas pueden combinarse entre sí ${ }^{(2,3)}$ (figura 2):

1. Fractura de Le Fort I o de trazo horizontal o fractura de Güerin.

2. Fractura de Le Fort II o de trazo piramidal.

3. Fractura de Le Fort III o disyunción cráneo-facial.

Las fracturas de Le Fort son producto de traumatismos severos, muy intensos en la región anterior de la cara, generalmente consecuencia de accidentes de tránsito; siendo la más frecuente la de Le Fort II. Al recibir todo el impacto en la región anterior de la cara se produce el trazo de fractura bilateral con la conminución respectiva de todo el segmento anterior que recibió el impacto. Con mayor frecuencia se observa esto en las fracturas de Le Fort II y III.

La fractura de Le Fort I dibuja un trazo horizontal, es transversal en la parte baja del tercio medio facial, parte de la espina nasal anterior y se dirige hacia la tuberosidad del maxilar superior (o borde posterior) bilateralmente pasando por todo el reborde alveolar y paladar, llegando incluso a las apófisis pterigoides o procesos pterigoideos ( $\mathrm{fi}$ gura 3 ).

La fractura de Le Fort II dibuja un trazo piramidal que parte de la unión fronto-nasal y desciende por la cara interna de ambas órbitas hacia la porción antral de los maxilares, en forma oblicua, hasta terminar posteriormente a nivel de la tuberosidad del maxilar superior de ambos lados, llegando incluso a los procesos pterigoideos (figura 4).

La fractura de Le Fort III o disyunción cráneo-facial es el trazo más alto del tercio medio facial y se inicia también a nivel fronto-nasal, dirigiéndose transversalmente y a través de ambas órbitas hacia las uniones fronto-malares, llegando incluso a los arcos cigomáticos, produciéndose así la separación o

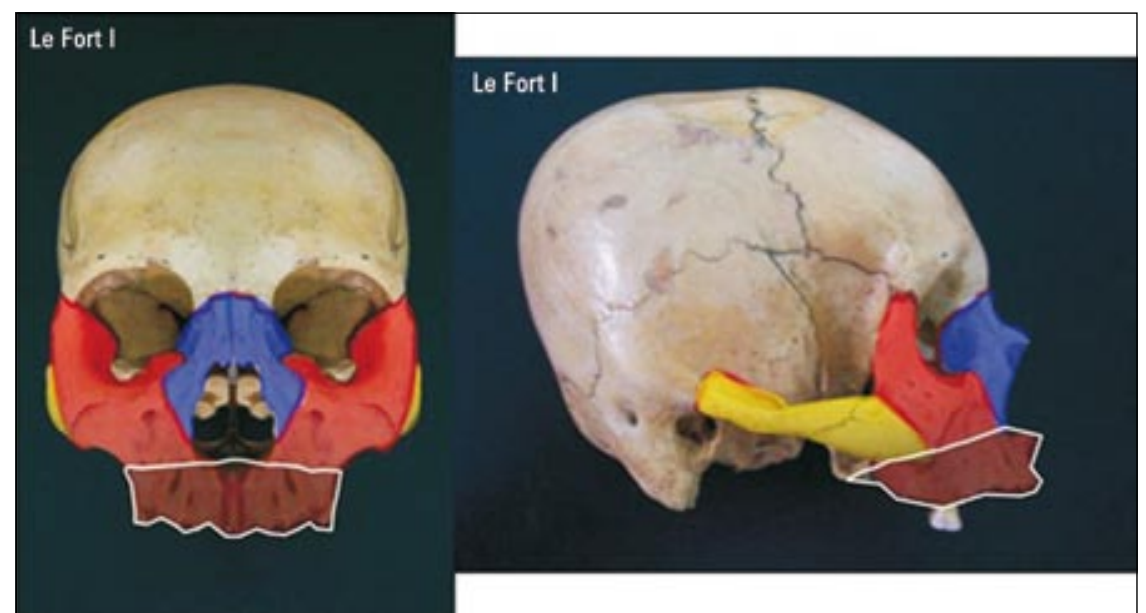

Figura 3: Trazo o área de fractura Le Fort I en color blanco y su relación con los complejos óseos del tercio medio facial. Vista frontal y lateral.

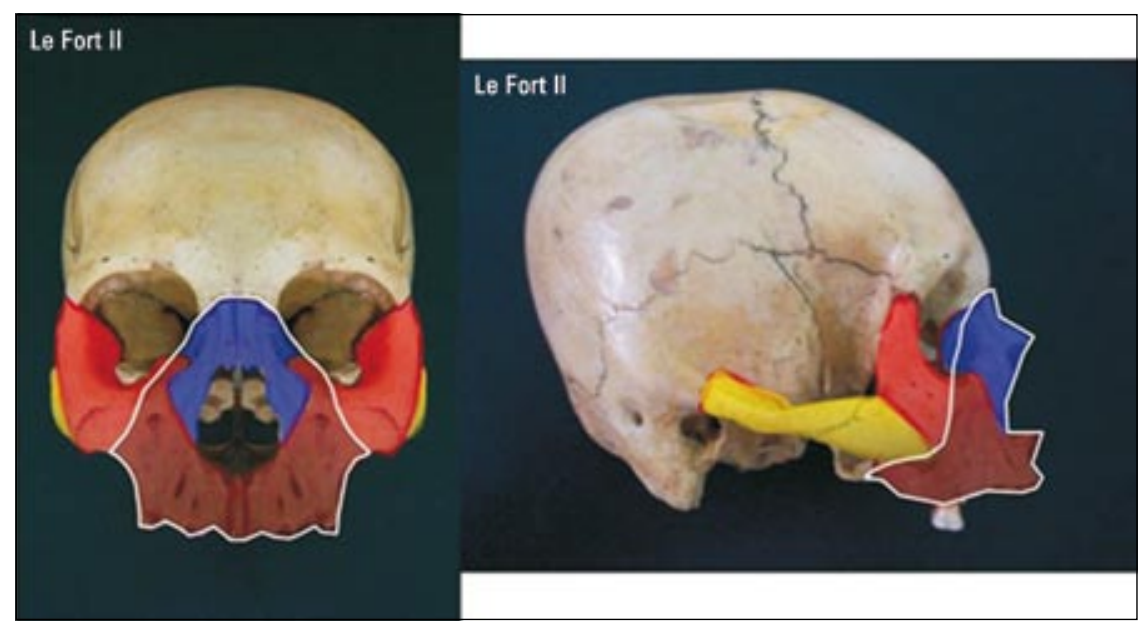

Figura 4. Trazo o área de fractura Le Fort II en color blanco y su relación con los complejos óseos del tercio medio facial. Vista frontal y lateral.

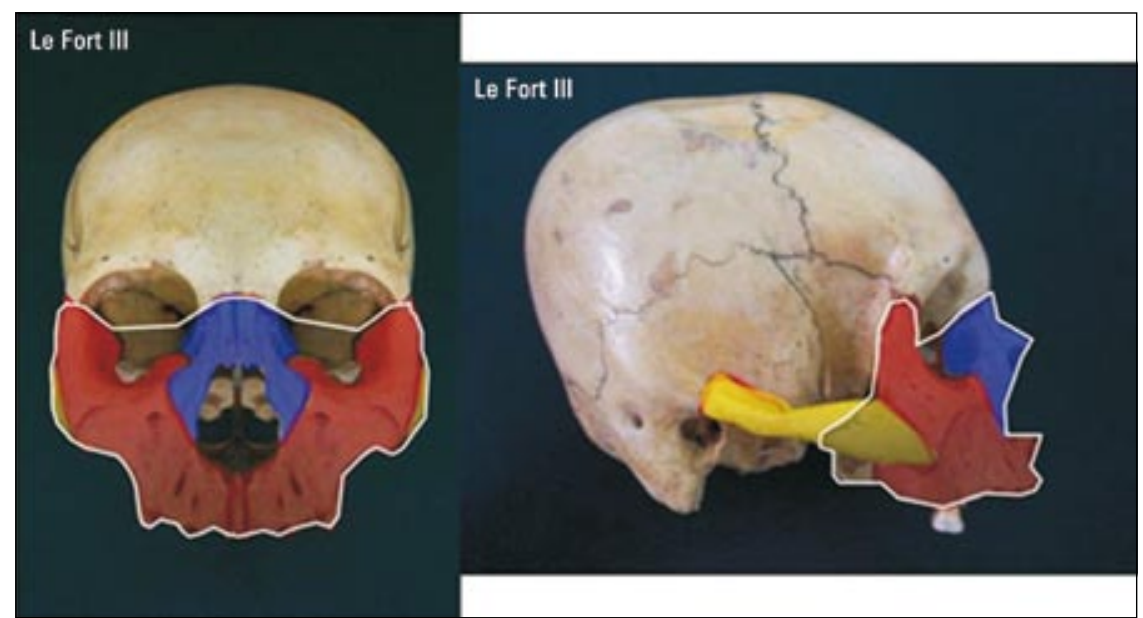

Figura 5. Trazo o área de fractura Le Fort III en color blanco y su relación con los complejos óseos del tercio medio facial. Vista frontal y lateral. 
disyunción entre el tercio medio facial y la base del cráneo; esta fractura es la más seria y compleja, produce mayor compromiso encéfalo-craneano y ocular, y puede cursar con la sección de uno o ambos nervios ópticos (figura 5).

Las fracturas de Le Fort tienen puntos o zonas en común entre sí: la fractura de Le Fort I con la de Le Fort II a nivel de la tuberosidad maxilar del lado derecho e izquierdo, mientras que entre la de Le Fort II con la de Le Fort III es a nivel de la unión fronto-nasal.

Podemos encontrar relación entre las áreas de fracturas de Le Fort y los complejos faciales mencionados en las fracturas de trazo unilateral. Así tenemos que el área de fractura Le Fort I está limitado a una porción del complejo máxilo-malar bilateralmente; esta corresponde al reborde alveolar en su totalidad (figura 3). El área de fractura Le Fort II comprende al complejo nasomaxilar y a la mayor parte del complejo máxilo-malar bilateralmente (figura 4). El área de fractura Le Fort III comprende al complejo naso-maxilar, al complejo máxilo-malar y prácticamente a la mitad del complejo témporo-malar bilateralmente (figura 5).

Generalmente, las fracturas del tercio medio facial son cerradas, siendo algunas veces parte de una combinación de varios elementos óseos fracturados. El diagnóstico se basa en el cuadro clínico, el mismo que varía según la estructura ósea afectada. Se tendrá en cuenta la edad del paciente y los signos locales inherentes a todo traumatismo, como el hematoma y el edema facial. Se confirmará mediante estudios radiológicos por imágenes, como radiografías del maxilar superior, senos paranasales (Watters, Hirz y Cadwell), órbitas, base de cráneo, tomografía axial computarizada (TAC) o tomografía espiral multicorte con reconstrucción tridimensional (TEM 3-D) ${ }^{(2,3)}$. Las imágenes tomográficas son las que nos dan en la actualidad una mayor claridad para visualizar el trazo o los trazos de fractura, permitiéndonos con ello una mayor precisión en el diagnóstico, lo que a su vez nos ayuda para la elección del material de osteosíntesis adecuado, razón por la que se prefiere iniciar el estudio radiológico con las imágenes tomográficas respectivas. En otras ocasiones, las tomografías nos permiten descartar fracturas donde las radiografías simples no lo permiten, ya que en estas últimas hay superposición de imágenes y en algunas circunstancias pueden mostrar una imagen sugestiva de fractura donde no la hay.

Las secuelas y complicaciones de estas fracturas están condicionadas a diversas circunstancias, teniendo en cuenta la edad del paciente. Se menciona la demora en el tratamiento, una mala técnica empleada, una mala colaboración del paciente (especialmente en los niños) y a una falla en la consolidación por la falta de formación del callo óseo (seudoartrosis), sobre todo en pacientes de edad avanzada, desnutridos, con alguna enfermedad sistémica concomitante o con un cuadro infeccioso local (osteomielitis y otros) ${ }^{(3)}$.

Las fracturas máxilo-faciales se presentan con una mayor incidencia en el sexo masculino, siendo el origen más frecuente los accidentes automovilísticos y las agresiones físicas, afectando mayormente a individuos entre 20 y 40 años de edad ${ }^{(2,3)}$.

Las fracturas del tercio medio facial pueden presentar compromiso encéfalo-craneano por la intensidad y vecindad del trauma, especialmente en las de Lefort II y III.

\section{MÉTODOS}

Se revisó las historias clínicas de pacientes portadores de fractura del tercio medio facial, desde junio de 1999 hasta mayo de 2009, en el Servicio de Cirugía de Cabeza, Cuello y Máxilo-Facial del Hospital Nacional Dos de Mayo.

La recolección de datos se hizo mediante una ficha (previamente elaborada), con información necesaria para la investigación (fracturas tercio medio, edad, sexo, agente causal y tipo de frac- tura), teniendo como finalidad determinar su frecuencia y clasificación.

El estudio realizado fue de tipo observacional descriptivo. Los datos obtenidos fueron revisados, clasificados y tabulados estadísticamente, en forma manual-electrónica, los mismos que son presentados en tablas (método de asociación), con la ayuda de programas de computación (Microsoft Word-Excel).

\section{RESULTADOS}

El total de historias clínicas revisadas de pacientes con 'fractura del tercio medio facial' fue 471 , de las cuales $88 \%$ correspondió a trazo unilateral y $12 \%$ a trazo bilateral o de Le Fort.

El 83\% correspondió solamente a fracturas del tercio medio; en $11 \%$ se acompañaron de fracturas del tercio inferior y en $6 \%$ de fracturas del tercio superior.

El $83 \%$ de los pacientes fue de sexo masculino y $17 \%$ femenino. La edad de presentación fue desde los 9 hasta los 80 años; $72,5 \%$ tuvo entre 21 y 40 años de edad (tabla 1). En $48 \%$ se debió a accidentes de tránsito (choque automovilístico y atropellos), 25\% a agresiones por robo (objeto contuso y proyectil arma de fuego), $15 \%$ fue casual (caídas, juegos, agresión animal, proyectil arma de fuego) y $12 \%$ peleas (tabla 1 ).

De la frecuencia según 'trazo de fractura y sexo' (tabla 2), podemos derivar que en las fracturas de trazo unilateral el $82 \%$ fue de sexo masculino y $18 \%$ de sexo femenino, mientras que en las fracturas de trazo bilateral $91 \%$ fue de sexo masculino y $9 \%$ de sexo femenino.

En las fracturas de trazo unilateral, se tuvo pacientes con fractura solamente a nivel del complejo máxilo-malar, en $74 \%$ de los casos, en el complejo naso-maxilar en $5 \%$ y en el témporomalar también en $5 \%$. Pacientes fracturados tanto en el complejo máxilo-malar como en el naso-maxilar juntos en $8 \%$, en el complejo máxilo-malar con el témporo-malar juntos 7\% y pacientes con los tres complejos fracturados, en 
Tabla 1. Fracturas del tercio medio facial ( 471 casos): frecuencia en porcentaje según edad, causa y sexo.

\begin{tabular}{|c|c|c|c|}
\hline & \multicolumn{2}{|c|}{ Sexo } & \multirow{2}{*}{$\begin{array}{c}\text { Tota } \\
(\%)\end{array}$} \\
\hline & Femenino (\%) & Masculino (\%) & \\
\hline \multicolumn{4}{|l|}{ Edad (en años) } \\
\hline 1 a 10 & 0,2 & 0,6 & 0,8 \\
\hline 11 a 20 & 0,9 & 2,5 & 3,4 \\
\hline 21 a 30 & 6,2 & 32,9 & 39,1 \\
\hline 31 a 40 & 4,9 & 28,5 & 33,4 \\
\hline 41 a 50 & 2,1 & 9,3 & 11,4 \\
\hline 51 a 60 & 1,9 & 7,2 & 9,1 \\
\hline 61 a 70 & 0,4 & 1,1 & 1,5 \\
\hline 71 a 80 & 0,4 & 0,9 & 1,3 \\
\hline Total (\%) & 17 & 83 & 100 \\
\hline \multicolumn{4}{|l|}{ Causa } \\
\hline Acc. de tránsito & 9 & 39 & 48 \\
\hline Robos & 4 & 21 & 25 \\
\hline Casual & 3 & 12 & 15 \\
\hline Peleas & 1 & 11 & 12 \\
\hline Total (\%) & 17 & 83 & 100 \\
\hline
\end{tabular}

$1 \%$ de los casos. En general, el complejo máxilo-malar fue afectado en $90 \%$ de los casos, el naso-maxilar en $14 \%$ y el témporo-malar en $13 \%$.

En las fracturas de trazo bilateral se encontró una mayor frecuencia para la Le Fort II, con $58 \%$ de los casos, mientras que la Le Fort I tuvo 26\% y la Le Fort III 16\%.

\section{DISCUSIÓN}

Los resultados obtenidos prácticamente coinciden con la literatura consultada y con trabajos elaborados anteriormente. Las fracturas del tercio medio facial son las más frecuentes de la región, pu-

Tabla 2. Frecuencia en porcentaje según trazo de fractura y sexo.

\begin{tabular}{cccc} 
Trazo de Fractura & \multicolumn{2}{c}{ Sexo } & $\begin{array}{c}\text { Total } \\
(\%)\end{array}$ \\
\cline { 2 - 3 } Unilateral & Femenino $(\%)$ & Masculino (\%) & 88 \\
Bilateral & 16 & 72 & 12 \\
Total (\%) & 1 & 11 & 100 \\
\hline
\end{tabular}

de arma de fuego; estas son causas que van en aumento, como consecuencia de la violencia en la que actualmente vivimos, tanto delictiva como del tránsito vehicular.

En las fracturas de trazo unilateral, el complejo máxilo-malar es el más afectado, solo o acompañado de fracturas del complejo naso-maxilar y/o del témporo-malar, teniendo en cuenta que las fracturas del complejo nasomaxilar (pirámide nasal) aisladas son vistas mayormente por el servicio de otorrinolaringología, las mismas que si son tomadas en consideración cambiarían la mayor incidencia obtenida en el presente trabajo. En las fracturas de trazo bilateral, la fractura de Le Fort II es la de mayor incidencia. El diagnóstico clínico depende de la estructura ósea afectada y se confirma mediante los estudios radiológicos respectivos, ayudando también a la elección del material de osteosíntesis. En muchas ocasiones, el manejo de estos pacientes se debe hacer conjuntamente con Neurocirugía y Oftalmología, debido al compromiso encéfalo-craneano y óculo-orbitario, respectivamente, producto de la intensidad y de la localización del traumatismo. Se debe actuar en forma oportuna para evitar las secuelas y complicaciones de estas fracturas, teniendo en cuenta la edad del paciente, demora en el tratamiento, no colaboración del paciente (especialmente en los niños) y a una falla en la consolidación ósea, sobre todo en pacientes de edad avanzada, desnutridos, con alguna enfermedad sistémica concomitante o infecciosa.

Debemos tener presente que contamos con gran parte de nuestra población que carece de recursos económicos o que es informal, con un problema serio de atención de salud, por no contar con algún tipo de seguro; incluso, en muchos de ellos las necesidades básicas están insatisfechas, con una estructura familiar deteriorada y viven en condiciones de hacinamiento. Por lo general, son personas que están subempleadas o desempleadas, lo que nos limita principalmente en la disponibilidad de 
imágenes radiográficas adecuadas y en la adquisición del material necesario para cada tipo de fractura, con la consiguiente demora diagnóstica, retraso en el tratamiento y secuelas respectivas.

Podemos concluir diciendo que las fracturas del tercio medio facial son las más frecuentes de la región facial y que su diagnóstico clínico dependerá de la estructura ósea afectada. Debemos actuar oportunamente para evitar así las secuelas y complicaciones propias de estas fracturas. Los accidentes de tránsito y los robos son las causas más frecuentes y van en aumento. Se espera que este trabajo ayude en la elaboración de protocolos de atención en los servicios de emergencia, dependiendo directamente del tipo de fractura, la edad y el agente causal.

\section{REFERENCIAS BIBLIOGRÁFICAS}

1. Avello F, Avello A. Nueva clasificación de las fracturas de trazo unilateral del tercio medio facial. An Fac med. 2007;68(1):75-9.

2. Avello A. Cirugía de Cabeza, del Cuello y Maxilofacial. Lima: Ed. MAD Corp S.A. 2002.

3. Avello F. Epidemiología y Clasificación de las Fracturas Máxilo-Faciales. Hospital Nacional Dos de Mayo. Tesis de especialidad. Lima: UNMSM. 2002.

4. Loré J. Atlas de Cirugia de Cabeza y Cuello. $3^{\text {a }}$ edición. Ed Panamericana. 1990.
5. Infante P, Garcia A, Infante M, Mayorga F, Espin F. Fracturas del tercio medio facial que implican a la órbita. Estudio retrospectivo de 208 fracturas en 163 pacientes. Rev Esp de Cir Oral y MáxiloFacial. 1999;21:1.

6. Weisman RA, Savino PJ. Management of patients with facial trauma and associated ocular-orbital injuries. Otolaryngol Clin North Amer. 1991;24:37.

7. De Man K, Wijngaarde R, Hes J, De Jong PL. Influence of age on the management of Blow-out fractures of the orbital floor. Int J Oral Maxilofac Surg. 1991;20(6):330.

8. Bakhmudov BR. The seasonal patterns of surgical morbidity of the maxillofacial area under the climatic conditions of the city of Derbent. Stomatologia (Mosk). 1997;76(1):60-3.

9. Leipziger LS, Manson PN. Nasoethmoid orbital fractures. Current concepts and management principles. Clin Plast Surg. 1992;19:167-93.

10. Knight JS, North JF. The classification of malar fractures: an analysis of displacement as a guide to treatment. Br J Plast Surg. 1961;13:325-32.

11. Garcia-Roco O. Fracturas orbitocigomáticas, valor de la clasificación de Knight y North en su terapéutica. Rev Cubana Estomatol. 2006;43(2). Disponible en: <http://scielo.sld. cu/scielo.php?script=sci_arttext\&pid $=$ S0034$75072006000200002 \&$ lng $=$ es\&nrm=iso $>$.

12. Manson PN, Markowitz B, Mirvis S, Dunham M, Yaremchuk M. Toward CT-based facial fracture treatment. Plast Reconstr Surg. 1990;85(2):20212.

13. Merino C, Albornoz C, Siré A, Queipo G. Fractura bilateral del arco cigomático, a propósito de un caso. Rev Archivo Médico de Camagüey. Cuba. 2005;9(6). Disponible en: http://redalyc.uaemex. $\mathrm{mx} / \mathrm{src} /$ inicio/ArtPdfRed.jsp?iCve $=211117924011$

14. Honig, JF, Mesten HA. Classification system and treatment of zygomatic arch fractures in the clinical setting. J Craniofac Surg. 2004;15(6):986-9.

15. Gruss JS. Naso-ethmoid-orbital fractures: Classification and role of primary bone grafting. Plast Reconstr Surg. 1985;75:303-15.
16. Zingg M, Laedrach K, Chen J, Chowdhury K, Vuillemin $T$, et al. Classification and treatment of zygomatic fractures: a review of 1025 cases. J Oral Maxillofac Surg. 1992;50(8):778-90.

17. Gruss JS, Wyck LV, Phillips JH, Antonyshyn O. The importance of the zygomatic arch in complex mid facial fracture repair and correction of post-traumatic orbitozygomatic deformities. Plast Reconstr Surg. 1990;85:878.

18. Manson PN. Traumatismos de la cara. En: Mc Carthy Joseph G, editor. Cirugía plástica: La cara I. Ed Panamericana. 1992;1-268.

19. Güerrissi J. Fracturas de cavidades orbitarias, tácticas y técnicas de tratamiento. Buenos Aires, Argentina: Ed AMOLCA. 2006.

20. Gruss JS, Buback PJ. Craniofacial fractures: an algorithm to optimize results. Clin Plast Surg. 1992;19:195-206.

21. Cuenca J, Álvarez C. Fracturas faciales complejas. Cir Plást. México DF. 2004;14(3):132-40.

Trabajo ganador del premio otorgado a la mejor Investigación en Cirugía durante la "XII Premiación Nacional de Trabajos de Investigación en Salud 2010"; Ministerio de Salud, Hospital Nacional Dos de Mayo, $135^{\circ}$ Aniversario. Lima, 26 de Febrero del 2010.

Trabajo recibido el 24 de octubre de 2012 y aceptado para publicación el 12 de diciembre de 2012.

\section{Correspondencia:}

Dr. Francisco Avello Canisto

Servicio de Cirugía de Cabeza, Cuello y MáxiloFacial,

Hospital Nacional Dos de Mayo. Parque de la Medicina.

Av. Grau s/n (altura cuadra 13), Barrios Altos.

Lima 1, Perú.

Correo-e:favelloc@hotmail.com 